\title{
Caracterización neuropsicológica de las quejas de memoria en la población general: relación con la sintomatología prefrontal y el estrés percibido
}

\author{
José M. Ruiz-Sánchez de León ${ }^{1,2,}$, Eduardo J. Pedrero-Pérez ${ }^{3}$ y Paz Lozoya-Delgado ${ }^{4}$ \\ ${ }^{1}$ Dpto. Psicología Básica II (Procesos Cognitivos). Universidad Complutense de Madrid \\ ${ }^{2}$ Centro de Prevención del Deterioro Cognitivo. Instituto de Salud Pública. Madrid Salud (Aynntamiento de Madrid) \\ ${ }^{3}$ Centro de Atención a Drogodependientes CAD 4 (San Blas). Instituto de Adicciones. Madrid Salud (Ayuntamiento de Madrid) \\ ${ }^{4}$ Centro de Tratamiento de la Lesión Cerebral LESCER
}

\begin{abstract}
Resumen: En los últimos años se ha relacionado la presencia de quejas cognitivas en los adultos jóvenes con perfiles atencionales y ejecutivos deficitarios, aunque no patognomónicos, que parecen justificar la aparición de los errores cotidianos. El presente estudio pretende analizar si la aparición de dichas quejas y su magnitud están relacionadas con un perfil neuropsicológico levemente inatento y disejecutivo. Por otro lado, se estudia si dichas quejas están también relacionadas con incrementos del estrés percibido, en la medida en que dichos incrementos pudieran funcionar como desencadenantes de las quejas. Para ello, se administró una batería de exploración neuropsicológica junto con el Cuestionario de Fallos de Memoria de la Vida Cotidiana, el Cuestionario Disejecutivo y la Escala de Estrés Percibido a una muestra de 99 individuos de población no clínica. Los resultados sugieren que los individuos de la población general que presentan quejas cognitivas presentan, por un lado, un menor rendimiento atencional, mnésico y ejecutivo y, por otro, una mayor sintomatología de tipo prefrontal. Se sugiere que este perfil cognitivo les hace más propensos a que los incrementos en el estrés percibido incrementen también dichos déficits, que son percibidos como alteraciones mnésicas sin que en sentido estricto lo sean.

Palabras clave: Quejas de memoria; memoria; metamemoria; atención; funciones ejecutivas; síndrome disejecutivo; estrés percibido; afrontamien-
\end{abstract} to.

\section{Introducción}

En los últimos años se han venido analizando algunas razones que pudieran explicar por qué determinados individuos de la población general refieren quejas de memoria en algún momento de sus vidas. Habitualmente la aparición de dichas quejas se relaciona con trastornos afectivos, como la depresión (Crane, Bogner, Brown y Gallo, 2007; Montejo, Montenegro, Fernández y Maestú, 2011), determinados perfiles de la personalidad (Pearman y Storandt, 2004; Verma, Pershad, Nehra, Kaur y Bhagat, 1996) o la calidad de vida autopercibida (Montejo, Montenegro, Fernández y Maestú, 2012; Pearman, 2009). Sin embargo, la mayoría de estos trabajos estudian poblaciones mayores y tratan de relacionar directamente las quejas con la probabilidad de desarrollar deterioro cognitivo asociado a enfermedades degenerativas (Schmand, Jonker, Hooijer y Lindeboom, 1996). Así, no parecen proporcionar suficiente información sobre los factores predisponentes y desencadenantes de las quejas cognitivas en la población menor de 65 años, en los que los estudios clínicos etiológicos y la exploración neuropsicológica resultan con

* Dirección para correspondencia [Correspondence address] José María Ruiz Sánchez de León (Buzón 119). Dpto. Psicología Básica II (Procesos Cognitivos). Universidad Complutense de Madrid. 28223, Pozuelo de Alarcón, Madrid (España). E-mail: jm.ruiz.sdl@gmail.com
Title: Neuropsychological characterization of memory complaints in the general population: relationship to prefrontal symptoms and perceived stress.

Abstract: In recent years, the presence of cognitive complaints in young adults has been related with attentional and executive deficit profiles, although not pathognomonic, which seem to justify the occurrence of everyday errors. This study aims to analyze whether the occurrence of such complaints and their magnitude are associated with a slightly dysexecutive and inattentive neuropsychological profile. On the other hand, we study whether these complaints are also related to increases in perceived stress, so that such increases could function as triggers of complaints. A neuropsychological battery and Memory Failures Everyday, Dysexecutive Questionnaire and Perceived Stress Scale were administered to a sample of 99 nonclinical participants. The results suggest that individuals in the general population who have cognitive complaints show, first, lower attentional, mnemonic and executive ratings and, second, increased prefrontal-type symptoms. It is suggested that this cognitive profile makes them more prone to increase these deficits due to increases in perceived stress, which are perceived as memory impairment without strictly being it.

Key words: Memory complaints; memory; metamemory; attention; executive functions; dysexecutive syndrome; perceived stress; coping.

frecuencia normales, y en los que la probabilidad de desencadenar una demencia a corto o medio plazo es anecdótica.

Uno de los factores predisponentes a las quejas cognitivas en los adultos jóvenes que ha comenzado a ser estudiado es la presencia de déficits atencionales y ejecutivos, no patognomónicos, que podrían justificar la aparición de errores cotidianos - en forma de despiste u olvidos - que no dependerían de una alteración mnésica per se (Ruiz, Llanero, Lozoya, Fernández y Pedrero, 2010). Efectivamente, existe una estrecha relación entre el funcionamiento de la atención y las funciones ejecutivas, y el funcionamiento de la memoria ( $\mathrm{Ti}$ rapu y Muñoz, 2005). Tanto desde un punto de vista teórico como aplicado, resulta plausible pensar que los individuos con menor habilidad para la gestión atencional superior, la inhibición de respuestas automáticas o la planificación, entre otros procesos, presenten a su vez menores habilidades mnésicas, en la medida en que las últimas dependen de las primeras. En esa línea, por ejemplo, los resultados en población general muestran relaciones significativas entre el rendimiento mnésico a largo plazo y el de la memoria de trabajo $(r=$ $.41 ; p<.001)$, el de atención alternante $(r=-.54 ; p<.001)$ o el de inhibición de respuestas motoras $(r=-.39 ; p<.001)$, entre otras (para consultar el patrón de correlaciones; Ruiz et al., 2010). En esta línea, se ha evidenciado cómo los individuos de entre 18 y 64 años que acuden a la consulta informando de problemas de memoria presentan, sin etiología que lo justifique, un rendimiento mnésico, atencional y ejecu- 
tivo inferior al de los individuos sin quejas. Estos déficits, aun cuando se encuentran dentro de la normalidad estadística, darían cuenta de los fallos de memoria que motivan la consulta.

El perfil neuropsicológico de los individuos con quejas de memoria no sólo presenta dichos déficits atencionales y ejecutivos, sino que además se observa en ellos una tendencia a presentar mayor sintomatología cotidiana relacionada con el funcionamiento de la corteza prefrontal (Lozoya, Ruiz y Pedrero, 2012). Se ha descrito que entre el 6\% y el $26 \%$ de los individuos sanos, sin ningún diagnóstico neurológico ni psiquiátrico, presenta dichos síntomas (Llanero et al., 2008; Pedrero et al., 2009; 2011); que se traducen en inatenciones esporádicas, respuestas desinhibidas o impulsivas, planes de acción deficitarios, mala supervisión de los mismos y problemas para generar alternativas a dichos planes. De nuevo, en la línea de lo propuesto, también es importante destacar la estrecha relación encontrada $(r=.65, p<.001)$ entre dicha sintomatología prefrontal y la presencia de quejas cognitivas (para consultar el patrón de correlaciones; Lozoya et al., 2012).

Así como la corteza prefrontal es también responsable de la estimación de la eficacia de las capacidades cognitivas (Chua, Schacter y Sperling, 2009; Jurado, Junqué, Vendrell, Treserras y Grafman, 1998; Kikyo, Ohki y Miyashita, 2002; Schyner et al., 2004; Vilkki, Servo y Surma-Aho, 1998; 1999), resulta plausible que los déficits del rendimiento prefrontal provoquen, además de los errores cotidianos, una deficitaria percepción de los mismos que conlleva la aparición de las quejas. Esta última idea está íntimamente relacionada con el concepto de metamemoria (Flavell, 1979; Nelson y Narens, 1990) y con las oscilaciones en la conciencia de los déficits que presentan los pacientes con lesiones que afectan a la corteza prefrontal (Pia, Neppi-Modona, Ricci y Berti, 2004; Rosen, 2011). Así, un perfil neuropsicológico levemente inatento y disejecutivo, junto con la presencia de sintomatología prefrontal, emergen como posibles factores predisponentes de interés en el estudio de la aparición de las quejas cognitivas en los adultos jóvenes.

Por otro lado, se ha hipotetizado acerca de la relación entre estos aspectos con el estrés percibido y la calidad de su afrontamiento (Lozoya et al., 2012; Ruiz et al., 2010). Los individuos peor dotados de habilidades mnésicas, atencionales y ejecutivas, podrían acabar presentando dificultades para adaptarse a los cambios por una mala gestión del estrés (Goldwater et al., 2009; Liston et al., 2006). De hecho, cuando aparece un estresor y es percibido como tal, el individuo debe poner en marcha un conjunto de habilidades para resolver su situación y, en esa medida, debe disponer de las capacidades cognitivas necesarias para llevarlas a cabo. También se ha evidenciado que el estrés crónico puede acabar afectando a la síntesis de catecolaminas y modular el rendimiento prefrontal (Arnsten y Li, 2005), por lo que resulta razonable pensar que los incrementos en el estrés percibido pudieran funcionar como desencadenantes de las quejas; en especial, en aquellos individuos peor dotados de habilidades cognitivas superiores, en la medida en que las demandas del medio superan dichas capacidades.

El presente estudio pretende analizar si, como sugieren los hallazgos previos, la aparición de quejas cognitivas y su magnitud están relacionadas con un perfil neuropsicológico levemente inatento y disejecutivo. Por otro lado, se estudia si dichas quejas están también relacionadas con un incremento del estrés percibido durante el último mes por parte de los individuos. De esa manera se podrán seguir formulando nuevas hipótesis acerca de los factores predisponentes y desencadenantes de las quejas en esta población, como cadena de antecedentes y consecuencias para, en última instancia, acercarnos a proponer intervenciones terapéuticas específicas, a día de hoy pobremente desarrolladas.

\section{Método}

\section{Participantes}

Se reclutó una muestra de 99 individuos de población no clínica (media de edad 34 años y 2 meses; DT =12 años y 9 meses; rango 18-64). Se trataba de 44 varones (media de edad 34 años y 2 meses; $D T=14$ años y 1 mes; rango 18-64) y 55 mujeres (media de edad 34 años y 2 meses; $D T=11$ años y 9 meses; rango 18-64) de los que el 5.1\% sólo contaban con estudios primarios $(4.5 \%$ de los varones, $5.5 \%$ de las mujeres), el $11.1 \%$ estudios de secundaria obligatoria $(9.1 \%$ varones, $12.7 \%$ mujeres), el $20.2 \%$ estudios de secundaria posobligatoria $(20.5 \%$ varones, $20 \%$ mujeres) y el $63,6 \%$ estudios universitarios $(65.9 \%$ varones, $61.8 \%$ mujeres $)$.

\section{Instrumentos}

Se aplicó una modificación del Cuestionario de Fallos de Memoria de la Vida Cotidiana, de 30 ítems, basado en el Memory Everyday Failures (MFE) (Sunderland, Harris y Baddeley, 1984; Sunderland, Harris y Gleave, 1984), que explora las quejas subjetivas de memoria. Por otro lado, se administró el Cuestionario Disejecutivo (DEX; Wilson, Alderman, Burgess, Emslie y Evans, 1996), de 20 ítems, con el objeto de valorar la presencia de sintomatología cognitiva, conductual y emocional relacionada con el funcionamiento de la corteza prefrontal. Ambos cuestionarios han sido recientemente validados en población general española mostrando unas adecuadas propiedades psicométicas (MFE-30, Lozoya et al., 2012; DEX-Sp, Pedrero et al., 2011). En último lugar, se aplicó la Escala de Estrés Percibido (EEP; Cohen, Kamarck y Mermelstein, 1983), formada por 14 preguntas directas, que valora los niveles de estrés y control experimentados en el último mes por el sujeto. Esta última escala ha sido también utilizada con éxito en otros trabajos con población española (Pedrero y Olivar, 2010).

Por otro lado, se administró una batería de exploración neuropsicológica similar a la propuesta por Ruiz, Pedrero, Rojo, Llanero y Puerta (2011), concebida para valorar de manera relativamente exhaustiva los procesos atencionales, 
mnésicos y ejecutivos en poblaciones sin daño cerebral adquirido ni demencias degenerativas. En la Tabla 1 y la Tabla 2 de los resultados se exponen las pruebas seleccionadas, clasificadas en función del proceso cognitivo subyacente principal con el que están relacionadas.

\section{Procedimiento}

Tras cumplimentar unos datos sociodemográficos básicos (edad, sexo y nivel de estudios), se formuló a todos los participantes la pregunta directa y dicotómica "¿tiene usted problemas de memoria?", a la que debían responder mediante un "si" o un " $n o$ ". Esta pregunta, que suele formularse de esa misma manera en la práctica profesional, sirvió como introducción a los tres cuestionarios administrados. Posteriormente se administró la batería de pruebas neuropsicológicas de acuerdo con las normas de aplicación y los criterios de corrección propios de cada manual. Todas las pruebas fueron aplicadas por neuropsicólogos debidamente formados, en similares condiciones, con idéntico material, en dos sesiones de entre 45 y 60 minutos. Durante el transcurso de las pruebas se ofreció a todos los participantes la posibilidad de realizar un descanso para evitar el efecto de la fatiga sobre su rendimiento. Todos los participantes fueron informados del objetivo de la evaluación psicométrica y neuropsicológica, y firmaron voluntariamente su consentimiento informado.

Se estableció, como criterio de exclusión de la muestra, que la persona estuviera o hubiera estado en tratamiento por algún proceso neurológico o psiquiátrico o que mostrara signos de alguno de ellos (demencia primaria, enfermedades metabólicas, ictus, traumatismos craneoencefálicos, hidrocefalia normotensiva, encefalitis, antecedentes neuroquirúrgicos, historial de abuso de drogas). También se habría excluido a aquellos que hubieran obtenido puntuaciones que pudieran haberse interpretado como sospechosas o sugerentes de simulación, si bien no se encontró ningún caso que se ajustara a este supuesto.

\section{Análisis estadístico}

Se realizaron pruebas robustas de comparación de medias (Brown-Forsythe), puesto que el $N$ de los grupos a comparar era bajo y no todas las pruebas neuropsicológicas cumplían los criterios de normalidad. Se utilizó el estadístico $d$ de Cohen (transformado en coeficiente de correlación) para estimar el tamaño del efecto de las diferencias. Se estimó la significación de cada correlación, una a una, mediante la prueba $t$. Se realizaron pruebas de correlación lineal de orden cero y parciales, tras controlar el efecto de variables intervinientes. Se realizó un análisis de regresión lineal por pasos sucesivos, transformando en variables dummy las de carácter ordinal o discreto. Finalmente se probaron diversas soluciones del análisis de caminos (path analysis) para encontrar la que mostrara mejores indicadores de bondad de ajuste. Para los análisis se han utilizado los paquetes estadísticos SPSS
15.0 (con la adición de una sintaxis para la prueba d de Cohen) y AMOS 4.01.

\section{Resultados}

En primer lugar, se compararon las puntuaciones en las pruebas neuropsicológicas de quienes declararon tener problemas de memoria con las obtenidas por quienes no declararon padecerlas (Tablas 1 y 2 ).

Tabla 1. Prueba robusta de diferencia de medias entre los grupos con y sin quejas de memoria en los test neuropsicológicos que valoran atención y memoria.

\begin{tabular}{|c|c|c|c|c|}
\hline \multicolumn{2}{|c|}{ TEST DE ATENCIÓN Y MEMORIA } & \multicolumn{3}{|c|}{$\begin{array}{l}\text { Brown- } p \\
\text { Forsythe }\end{array}$} \\
\hline \multicolumn{2}{|c|}{ Atención se-Clave de números (WAIS) } & 4.79 & .036 & .26 \\
\hline \multirow{8}{*}{$\begin{array}{l}\text { lectiva y } \\
\text { sostenida }\end{array}$} & Test d 2 de atención & & & \\
\hline & Total de Respuestas & 1.48 & .229 & \\
\hline & Total de Aciertos & 0.77 & .386 & \\
\hline & Total de Omisiones & 0.03 & .871 & \\
\hline & Total de Errores & 1.63 & .216 & \\
\hline & TMT (Forma A) & & & \\
\hline & Tiempo de ejecución & 0.56 & .462 & \\
\hline & Errores & 0.54 & .467 & \\
\hline \multicolumn{5}{|c|}{ Atención di-TMT (Forma B) } \\
\hline \multirow{3}{*}{$\begin{array}{l}\text { vidida y } \\
\text { alternante }\end{array}$} & Tiempo de ejecución & 2.12 & .158 & \\
\hline & Errores & 1.01 & .325 & \\
\hline & Test Breve de Atención (TBA) & 1.62 & .211 & \\
\hline \multirow{4}{*}{$\begin{array}{l}\text { Amplitud } \\
\text { atencional y } \\
\text { memoria de } \\
\text { trabajo }\end{array}$} & Dígitos Directos (WMS-III) & 18.25 & .000 & .43 \\
\hline & Dígitos Inversos (WMS-III) & 10.97 & .002 & .34 \\
\hline & Letras y Dígitos (WMS-III) & 12.22 & .001 & .38 \\
\hline & Cambio de reglas (BADS) & 0.03 & .853 & \\
\hline \multirow{13}{*}{ Memoria } & Figura compleja de Rey & & & \\
\hline & Tiempo de Copia & 0.52 & .478 & \\
\hline & Puntuación de Copia & 1.04 & .318 & \\
\hline & Puntuación Memoria Inmediata & 3.27 & .079 & \\
\hline & Puntuación Memoria Demorada & 3.49 & .070 & \\
\hline & Lista de palabras (WMS-III) & & & \\
\hline & $1^{\mathrm{a}}$ Lectura & 2.35 & .133 & \\
\hline & Aprendizaje Total & 2.15 & .152 & \\
\hline & Memoria a Corto Plazo (MCP) & 2.27 & .143 & \\
\hline & Memoria a Largo Plazo (MLP) & 2.14 & .155 & \\
\hline & Intrusiones en MLP & 0.29 & .594 & \\
\hline & Reconocimiento & 1.96 & .174 & \\
\hline & Memoria Incidental CN (WAIS) & 5.62 & .024 & .29 \\
\hline
\end{tabular}

NOTA: $r d=$ Estimador d de Cohen del tamaño del efecto, transformado en coeficiente de correlación.

Se observan diferencias significativas entre los sujetos en función de si presentan o no quejas de memoria en pruebas de amplitud atencional y memoria de trabajo (dígitos directos e inversos y letras y números de la WMS-III; Wechsler, 1999b), la puntuación de inhibición del test de los cinco dígitos (Sedó, 2007), abstracción verbal (semejanzas de la WAIS; Wechsler, 1999a) y la velocidad de procesamiento (clave de números de la WAIS) junto con la medida de memoria incidental en esa misma última prueba. 
Tabla 2. Prueba robusta de diferencia de medias entre los grupos con y sin quejas de memoria en los test neuropsicológicos que valoran funciones ejecutivas.

\begin{tabular}{|c|c|c|c|c|}
\hline \multicolumn{2}{|c|}{$\begin{array}{l}\text { TEST DE FUNCIONES } \\
\text { EJECUTIVAS }\end{array}$} & \multirow[t]{2}{*}{$\begin{array}{l}\text { Brown- } \\
\text { Forsythe }\end{array}$} & \multirow[t]{2}{*}{$p$} & \multirow[t]{2}{*}{$r_{d}$} \\
\hline Inhibición & Test de Stroop & & & \\
\hline & Palabras & 1.88 & .179 & \\
\hline respuestas & Colores & 0.35 & .560 & \\
\hline automáti- & Palabras / Colores & 3.33 & .077 & \\
\hline \multirow[t]{9}{*}{ cas } & $\begin{array}{l}\text { Puntuación de Interferen- } \\
\text { cia }\end{array}$ & 3.83 & .059 & \\
\hline & Test de los cinco dígitos & & & \\
\hline & Lectura & 1.96 & .176 & \\
\hline & Conteo & 0.44 & .515 & \\
\hline & Elección & 0.10 & .750 & \\
\hline & Alternancia & 0.28 & .599 & \\
\hline & Puntuación de Inhibición & 5.45 & .025 & .27 \\
\hline & Puntuación de Flexibilidad & 0.55 & .465 & \\
\hline & Errores en Test de Hayling & 0.16 & .692 & \\
\hline \multirow{8}{*}{$\begin{array}{l}\text { Planifica- } \\
\text { ción }\end{array}$} & Torre de Hanoi & & & \\
\hline & Movs. con 3 Discos & 0.73 & .401 & \\
\hline & $\begin{array}{l}\text { Movs. Ilegales con } 3 \text { Dis- } \\
\text { cos }\end{array}$ & 0.07 & .789 & \\
\hline & Tiempo con 3 Discos & 1.15 & .292 & \\
\hline & Movs. con 4 Discos & 0.02 & .889 & \\
\hline & $\begin{array}{l}\text { Movs. Ilegales con } 4 \text { Dis- } \\
\text { cos }\end{array}$ & 1.06 & .312 & \\
\hline & Tiempo con 4 Discos & 0.84 & .368 & \\
\hline & $\begin{array}{l}\text { Estimación } \\
\text { (BADS) }\end{array}$ & 0.10 & .756 & \\
\hline \multirow{7}{*}{$\begin{array}{l}\text { Flexibili- } \\
\text { dad cogni- } \\
\text { tiva }\end{array}$} & Fluidez Verbal & & & \\
\hline & Fonológica $(\mathrm{F})$ & 2.17 & .148 & \\
\hline & Semántica (Animales) & 1.57 & .216 & \\
\hline & Alternante ( $\mathrm{P}+$ Nombres $)$ & 0.21 & .648 & \\
\hline & Test de los Cinco Puntos & & & \\
\hline & Fluidez de Diseños Total & 3.09 & .089 & \\
\hline & Perseveraciones & 0.22 & .644 & \\
\hline \multirow[t]{2}{*}{$\begin{array}{l}\text { Abstrac- } \\
\text { ción }\end{array}$} & progresivas & 1.38 & .251 & \\
\hline & Semejanzas (WAIS) & 4.48 & .044 & .27 \\
\hline
\end{tabular}

NOTA: $r d=$ estimador d de Cohen del tamaño del efecto, transformado en coeficiente de correlación.

Considerando a continuación la variable quejas como variable continua, a partir de las puntuaciones obtenidas en el MFE-30, se exploran las correlaciones observadas entre estas puntuaciones y las obtenidas en las pruebas neuropsicológicas. En cuanto al estrés percibido, y asumiendo la igualdad de varianzas (Levene $F=.09 ; p=.77$ ), no existen diferencias significativas entre quienes manifiestan quejas y quienes no lo hacen $\left(t_{.05 ; 97}=.22 ; p=.83\right)$. Sin embargo, sí aparecen correlaciones significativas entre la puntuación dimensional obtenida en el MFE-30 y las obtenidas en la EEP $(r=.37$; $t .05$; $\left.{ }_{96}=3.89 ; p<.001\right)$. Por ello, a continuación se estimaron nuevamente las correlaciones entre MFE-30 y las puntuaciones obtenidas en las pruebas neuropsicológicas, pero en esta ocasión controlando el estrés percibido (Tablas 3 y 4).

Así, existe una relación entre la puntuación en el MFE-30 y las pruebas neuropsicológicas, que muestran efectos significativos en pruebas de velocidad de procesamiento y memoria incidental (clave de numeros de la WAIS), aprendizaje y memoria verbal y visual (tanto en la lista de palabras de la WMSIII como en la figura compleja de Rey; Rey, 2009), memoria de trabajo (letras y numeros de la WMS-III; test breve de atención, Schretlen, Bobholz y Brandt, 1996; y cambio de regla de la BADS, Wilson et al., 1996), interferencia e inhibición de respuestas (denominación de colores en ensayos incongruentes del test de Stroop; Golden, 2005), medidas de planificación (estimación temporal de la BADS y movimiento ilegales en la torre de Hanoi; Shallice, 1982) y abstracción verbal y no verbal (semejanzas y matrices progresivas de la WAIS).

Para conocer el efecto de diversas variables sobre las quejas de memoria se efectuó un análisis de regresión lineal sobre las puntuaciones del MFE-30 y como variables predictoras el nivel académico, el sexo (variables dummy), la edad y el estrés percibido. Sólo esta última ofreció capacidad predictiva $\left(R^{2}=.136 ; \beta=.37 ; t=3.89 ; p<.001\right)$.

Se estudió también la relación entre las quejas de memoria, el estrés y las dificultades de funcionamiento en la vida diaria, estimadas mediante el DEX-Sp. Éste presentó una importante correlación con las puntuaciones del MFE-30 $(r$ $=.65 ; t=8.47 ; p<.0001)$, más con el componente de dificultades en el inicio de la conducta $(r=.70 ; t=9.57 ; p<$ $.0001)$ que con el de su interrupción $(r=.47 ; t=5.27 ; p<$ $.0001)$, aunque ambos con igual significación. También apareció una correlación importante entre el DEX-Sp y el estrés percibido medido por la EEP $(r=.43 ; t=4.72 ; p<.0001)$.

Por último, para conocer el sentido de las relaciones entre estas tres variables se efectuaron diferentes análisis de caminos (path análisis), encontrando que sólo uno de ellos alcanzó una adecuada bondad de ajuste $\left(\chi^{2}=1.524 ; g l=1\right.$; $C M I N / D F=1.524 ;$ RMSE $A=.08 ; N F I=.99 ;$ IFI = .99; $E C V I=.18-.26)$. El sentido de las relaciones entre estas tres variables sugiere que el estrés percibido explica el $28 \%$ de la varianza de la sintomatología frontal $\left(R^{2}=.28\right)$, y ésta a su vez el $59 \%$ de las quejas de memoria $\left(R^{2}=.59\right)$. 
Tabla 3. Correlaciones entre las puntuaciones del MFE-30 y las obtenidas en pruebas neuropsicológicas que valoran atención y memoria.

\begin{tabular}{|c|c|c|c|c|c|c|c|}
\hline \multicolumn{2}{|c|}{ TEST DE ATENCIÓN Y MEMORIA } & \multicolumn{3}{|c|}{ Correlaciones de orden cero } & \multicolumn{3}{|c|}{$\begin{array}{l}\text { Correlaciones parciales } \\
\text { (controlando estrés percibido) }\end{array}$} \\
\hline & & $r$ & $t$ & Sig. & $r$ & $t$ & Sig. \\
\hline \multirow{9}{*}{$\begin{array}{l}\text { Atención } \\
\text { selectiva y } \\
\text { sostenida }\end{array}$} & Clave de números (WAIS) & -.20 & 2.01 & $p<.05$ & -.18 & 1.8 & $p<.05$ \\
\hline & Test d2 de atención & & & & & & \\
\hline & Total de Respuestas & -.06 & & & & & \\
\hline & Total de Aciertos & -.04 & & & & & \\
\hline & Total de Omisiones & .09 & & & & & \\
\hline & Total de Errores & .07 & & & & & \\
\hline & TMT (Forma A) & & & & & & \\
\hline & Tiempo de ejecución & -.06 & & & & & \\
\hline & Errores & -.06 & & & & & \\
\hline \multirow{4}{*}{$\begin{array}{l}\text { Atención } \\
\text { dividida y } \\
\text { alternante }\end{array}$} & TMT (Forma B) & & & & & & \\
\hline & Tiempo de ejecución & .04 & & & & & \\
\hline & Errores & -.06 & & & & & \\
\hline & Test Breve de Atención (TBA) & -.24 & 2.44 & $p<.01$ & -.2 & 2.01 & $p<.05$ \\
\hline \multirow{4}{*}{$\begin{array}{l}\text { Amplitud } \\
\text { atencional y } \\
\text { memoria de } \\
\text { trabajo }\end{array}$} & Dígitos Directos (WMS-III) & -.06 & & & & & \\
\hline & Dígitos Inversos (WMS-III) & -.12 & & & & & \\
\hline & Letras y Dígitos (WMS-III) & -.21 & 2.16 & $p<.05$ & -.21 & 2.16 & $p<.05$ \\
\hline & Cambio de reglas (BADS) & .28 & 2.87 & $p<.01$ & .19 & 1.9 & $p<.05$ \\
\hline \multirow[t]{13}{*}{ Memoria } & Figura compleja de Rey & & & & & & \\
\hline & Tiempo de Copia & .01 & & & & & \\
\hline & Puntuación de Copia & .04 & & & & & \\
\hline & Puntuación Memoria Inmediata & -.18 & 1.8 & $p<.05$ & -.13 & & \\
\hline & Puntuación Memoria Demorada & -.17 & 1.7 & $p<.05$ & -.14 & & \\
\hline & Lista de palabras (WMS-III) & & & & & & \\
\hline & $1^{\mathrm{a}}$ Lectura & -.15 & & & & & \\
\hline & Aprendizaje Total & -.27 & 2.76 & $p<.01$ & -.31 & 3.21 & $p<.01$ \\
\hline & Memoria a Corto Plazo (MCP) & -.21 & 2.16 & $p<.05$ & -.2 & 2.01 & $p<.05$ \\
\hline & Memoria a Largo Plazo (MLP) & -.24 & 2.44 & $p<.01$ & -.26 & 2.69 & $p<.01$ \\
\hline & Intrusiones en MLP & .12 & & & & & \\
\hline & Reconocimiento & -.16 & & & & & \\
\hline & Memoria Incidental CN (WAIS) & -.24 & 2.44 & $p<.01$ & -.23 & 2.33 & $p<.05$ \\
\hline
\end{tabular}

Tabla 4. Correlaciones entre las puntuaciones del MFE-30 y las obtenidas en pruebas neuropsicológicas que valoran funciones ejecutivas. TEST DE FUNCIONES EJECUTIVAS Correlaciones de orden cero Correlaciones parciales (controlando estrés percibido)

\begin{tabular}{|c|c|c|c|c|c|c|c|}
\hline & & & & & (con & ando $c$ & és percibido) \\
\hline & & $\bar{r}$ & $t$ & Sig. & $r$ & $t$ & Sig. \\
\hline Inhibición de & Test de Stroop & & & & & & \\
\hline respuestas & Palabras & -.04 & & & & & \\
\hline automáticas & Colores & -.22 & 2.22 & $p<.05$ & -.23 & 2.33 & $p<.05$ \\
\hline & Palabras / Colores & -.22 & 2.22 & $p<.05$ & -.2 & 2.01 & $p<.05$ \\
\hline & Puntuación de Interferencia & -.16 & & & & & \\
\hline & Test de los cinco dígitos & & & & & & \\
\hline & Lectura & .19 & 1.91 & $p<.05$ & .13 & & \\
\hline & Conteo & .15 & & & & & \\
\hline & Elección & .09 & & & & & \\
\hline & Alternancia & .16 & & & & & \\
\hline & Puntuación de Inhibición & -.11 & & & & & \\
\hline & Puntuación de Flexibilidad & .08 & & & & & \\
\hline & Errores en Test de Hayling & -.03 & & & & & \\
\hline Planificación & Torre de Hanoi & & & & & & \\
\hline & Movs. con 3 Discos & .04 & & & & & \\
\hline & Movs. Ilegales con 3 Discos & .29 & 2.98 & $p<.01$ & .2 & 2.05 & $p<.05$ \\
\hline & Tiempo con 3 Discos & .19 & 1.91 & $p<.05$ & .11 & & \\
\hline & Movs. con 4 Discos & .13 & & & & & \\
\hline & Movs. Ilegales con 4 Discos & .13 & & & & & \\
\hline & Tiempo con 4 Discos & .12 & & & & & \\
\hline & Estimación temporal (BADS) & .29 & 2.98 & $p<.01$ & .27 & 2.81 & $p<.01$ \\
\hline
\end{tabular}




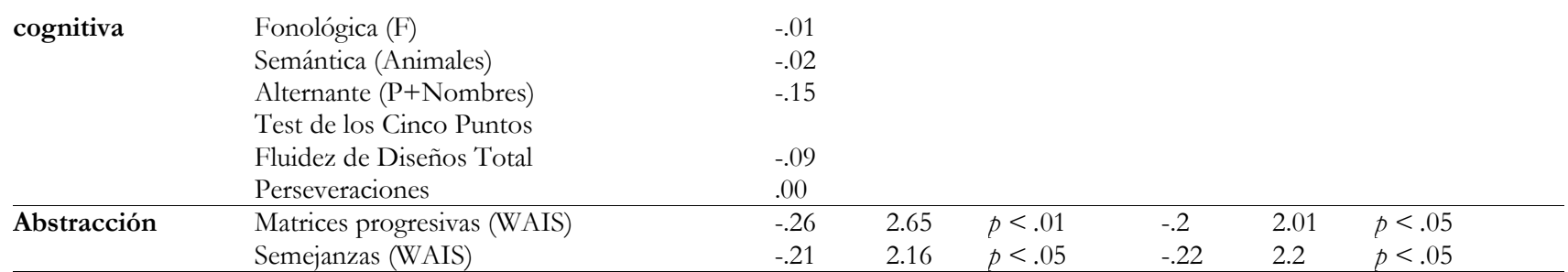

\section{Discusión}

Cuando se valora el rendimiento neuropsicológico de un paciente deben describirse, tanto cuantitativa como cualitativamente, las alteraciones en la codificación, el almacenamiento y el posterior recuerdo de la información; en especial cuando los síntomas referidos por él o por sus informadores cercanos hacen sospechar la presencia de un cuadro amnésico. Sin embargo, uno de los motivos de consulta más frecuentes en la práctica profesional diaria es la presencia de quejas de memoria en individuos en los que ni la exploración cognitiva ni el estudio etiológico es capaz de esclarecer la razón por la que aparecen dichas quejas (Ruiz et al, 2010). Este trabajo pretende analizar las estrechas relaciones que parecen mantener tres variables decisivas en la aparición de las quejas en la población general: el rendimiento neuropsicológico, la sintomatología prefrontal en la vida diaria y el estrés percibido.

Los resultados encontrados apoyan las hipótesis de partida en el sentido en que atendiendo a la variable dicotómica, sin quejas / con quejas de memoria, los individuos que sí las presentan obtienen puntuaciones inferiores en las medidas de velocidad de procesamiento, amplitud atencional, memoria de trabajo, inhibición y abstracción. Es interesante destacar que la única puntuación relativa al funcionamiento de la memoria en la que ambos grupos se diferencian es en la memoria incidental de la clave de números de la WAIS; en la medida en que la codificación de la información valorada con esa prueba no es intencional y, por tanto, depende en buena medida de las capacidades atencionales desplegadas durante la fase de estudio (Ruiz, Fernández y González, 2006). Profundizando en este hallazgo, se evidencia cómo los individuos con mayor puntuación en el MFE-30, obtienen también puntuaciones menores en esas mismas medidas - velocidad de procesamiento, amplitud atencional, memoria de trabajo, inhibición y abstracción - y, además, puntuaciones menores en las medidas de aprendizaje y memoria, tanto verbal como visual, y en la capacidad de planificación.

Respecto a la sintomatología de tipo prefrontal, los resultados muestran cómo tanto la puntuación total del DEX-Sp como sus dos factores, desorganización/apatía y desinhibición/impulsividad (Pedrero et al., 2011) correlacionan con la magnitud de las quejas; al igual que lo había hecho en estudios precedentes con diferentes muestras (Lozoya et al., 2012; Ruiz et al., 2010). Por otro lado, se evidencia la estrecha relación entre las quejas de memoria y el estrés percibido autoinformado por los participantes (EEP; Cohen et al.,
1983), siendo esta variable la única que ofrece una cierta capacidad predictiva sobre la aparición de dichas quejas; en contraste con las variables sociodemográficas que no se mostraron significativas.

Así como era relevante analizar la estrecha relación entre las puntuaciones de las tres medidas de autoinforme, el análisis de caminos ha mostrado que: i) el estrés tiene un cierto poder predictivo sobre la aparición de sintomatología prefrontal y, a su vez, ii) esta sintomatología tiene poder predictivo sobre la aparición y la magnitud de las quejas.

Respecto a la primera relación, existen evidencias de cómo el estrés agudo afecta a las tareas que requieren operaciones dependientes del córtex prefrontal (Arnsten, 1998). También se han realizado estudios en los que se muestra cómo los estresores sociales deterioran aspectos ejecutivos tales como la flexibilidad cognitiva (Alexander, Hillier, Smith, Tivarus y Beversdorf, 2007) o la memoria de trabajo (Luethi, Meier y Sandi, 2009). Otros trabajos han encontrado que la exposición a estrés evaluativo no afecta a las puntuaciones de la amplitud atencional (bucle fonológico valorado mediante dígitos directos de la WMS-III), pero sí a las puntuaciones que comprometen mecanismos superiores del ejecutivo central (digitos inversos de la WMS-III); lo que sugiere que, en efecto, el incremento del estrés condiciona el rendimiento de la memoria de trabajo (D’Esposito y Postle, 1999; Klein y Boals, 2001; Lewis, Nikolova, Chang y Weekes, 2008) y, en función de los resultados de este estudio, también a otros componentes ejecutivos y de la velocidad de procesamiento.

En cuanto a la segunda predicción del análisis de caminos, existen evidencias basadas en la relación que mantiene el funcionamiento de la corteza prefrontal y el concepto de metamemoria (Flavell, 1971; Nelson, 1996). Diversos estudios muestran la relación entre lesiones en los lóbulos frontales y deterioro en la metamemoria (Vilkki, et al., 1998; 1999); tanto en la fibromialgia (Pericot et al., 2009), en cuadros psicosomáticos (Metternich, Schmidtke y Hüll, 2009), la enfermedad de Alzheimer (Mimura, 2008) o la enfermedad de Parkinson (Baran, Tekcan, Gürvit y Boduroglu, 2009; Maruyama, 1997).

En definitiva, los resultados sugieren que los individuos de la población general que presentan quejas subjetivas de tipo cognitivo presentan, por un lado, un menor rendimiento atencional, mnésico y ejecutivo y, por otro, una mayor sintomatología de tipo prefrontal. A su vez, este perfil cognitivo les hace más propensos a que, cuando cambian las condiciones del medio y se incrementa su estrés percibido, se incrementen también sus déficits - traducidos en errores y despistes cotidianos - que son percibidos como alteraciones mné- 
sicas sin serlo. Esta misma relación, entendida como una relación circular, ha sido propuesta de manera similar por otros autores en el ámbito del envejecimiento, afirmando que las quejas de memoria influyen en las estrategias de afrontamiento y, ambas, en la calidad de vida; siendo la variable locus de control la variable antecedente más importante en los mayores (Potter, Hartman y Ward, 2009; Verhaeghen, Geraerts y Marcoen, 2000)

Por todo lo expuesto, deberían desarrollarse propuestas terapéuticas específicas basadas en estos hallazgos dado que, en la actualidad, los pocos trabajos orientados al tratamiento de las quejas cognitivas en adultos jóvenes se centran en la mera administración de tareas de estimulación cognitiva. Aunque este acercamiento produce algunos resultados modestos, sin duda resultan insuficientes. En efecto, la interven-

\section{Referencias}

Alexander, J. K., Hillier, A., Smith, R. M., Tivarus, M. E.Y. y Beversdorf, D. Q. (2007). Beta-adrenergic modulation of cognitive flexibility during stress. Journal of Cognitive Neuroscience, 19, 468-478.

Arnsten, A. F. T. The biology of feeling frazzled. Science, 280, 1711-1712

Arnsten, A. F. T. y Li, B. M. (2005). Neurobiology of executive functions: catecholamine influences on prefrontal cortical functions. Biological Psychiatry, 57, 1377-1384.

Baran, B., Tekcan, A. I., Gürvit, H., Boduroglu, A. (2009). Episodic memory and metamemory in Parkinson's disease patients. Neuropsychology, 23(6), 736-45

Chua, E. F., Schacter, D. L. y Sperling, R. A. (2009). Neural correlates of metamemory: a comparison of feeling-of-knowing and retrospective confidence judgments. Journal of Cognitive Neuroscience, 21, 1751-1765.

Cohen, S., Kamarck, T. y Mermelstein, R. A. (1983). Global measure of perceived stress. Journal of Health and Social Behaviour, 24, 385-396.

Conrad, A. y Roth, W. T. (2007). Muscle relaxation therapy for anxiety disorders: it works but how?. Journal of Anxiety Disorders, 21(3), 243-64. Epub 2006 Sep 1. Review

Crane, M. K., Bogner, H. R., Brown, G. K. y Gallo, J. J. (2007). The link between depressive symptoms, negative cognitive bias and memory complaints in older adults. Aging and Mental Health, 11, 708-715.

De Noreña, D., Ríos, M., Bombín, I., Sánchez, I., García, A. y Tirapu, J. (2010). Efectividad de la rehabilitación neuropsicológica en el daño cerebral adquirido (I): atención, velocidad de procesamiento, memoria y lenguaje. Revista de Neurología, 51, 687-98.

D'Esposito, M. y Postle, B. R. (1999). The dependence of span and delayed-response performance on prefrontal cortex. Neuropsychologia, 37, 1303-1315.

Flavell, J. H. (1971). First dicussant comments: Whats is memory development the development of?. Human Development, 14, 272-278.

Flavell, J. H. (1979). Metacognition and cognitive monitoring: A new area of cognitive development inquiry. American Psychologist, 34, 906-911.

Golden, C. J. (2005). Test de colores y palabras (Stroop). Madrid: TEA.

Goldwater, D. S., Pavlides, C., Hunter, R. G., Bloss, E. B., Hof, P.R., McEwen, B. S. y Morrison, J. H. (2009). Structural and functional alterations to rat medial prefrontal cortex following chronic restraint stress and recovery. Neuroscience, 164, 798-808.

González, A y Amigo, I. (2000). Efectos inmediatos del entrenamiento en relajación muscular progresiva sobre índices cardiovasculares. Psicothe$m a, 12(1), 25-32$.

Jurado, M. A, Junqué, C., Vendrell, P., Treserras, P. y Grafman, J. (1998). Overestimation and unreliability in "feeling-of-doing" judgements about temporal ordering performance: impaired self-awareness following frontal lobe damage. Journal of Clinical and Experimental Neuropsychology, 20, 353-364.

Kikyo, H., Ohki, K. y Miyashita, Y. (2002). Neural correlates for feeling of knowing. Neuron, 36, 177-186. ción psicológica en estos sujetos debería enfocarse concretamente a los posibles desencadenantes comentados: i) entrenamiento neuropsicológico en estrategias de mejora atencional, mnésica y ejecutiva adaptados para población general (De Noreña et al., 2010; Muñoz y Tirapu, 2004), y ii) programas de entrenamiento en habilidades de afrontamiento del estrés y resolución de problemas (Meichenbaum y Cameron, 1987). No obstante, y dada la complejidad de la variables intervinientes, podrían resultar interesantes otros acercamientos basados en la relajación (Conrad y Roth, 2007; González y Amigo, 2000) o el neurofeedback (Vernon et al., 2003), que ha mostrado incrementar tras pocas sesiones el rendimiento cognitivo y los mecanismos de afrontamiento del estrés en poblaciones no clínicas.

Klein, K. y Boals, A. (2001). The relationship of life event stress and working memory capacity. Applied Cognitive Psychology, 15, 565-579

Lewis, R., S., Nikolova, A., Chang, D. J. y Weekes, N. Y. (2008). Examination stress and components of working memory. Stress, 11(2), 108-114.

Liston, C., Miller, M. M., Goldwater, D. S., Radley, J. J., Rocher, A. B., Hof, P. R., Morrison, J. H. y McEwen, B. S. (2006). Stress-induced alterations in prefrontal cortical dendritic morphology predict selective impairments in perceptual attentional set-shifting. The Journal of Neurosience, 26, 7870-7874.

Llanero, M., Ruiz, J. M., Pedrero, E. J., Olivar, A., Bouso, J. C., Rojo, G., et al. (2008). Sintomatología disejecutiva en adictos a sustancias en tratamiento mediante la versión española del cuestionario disejecutivo (DEX-Sp). Revista de Neurología, 47, 457-463.

Lozoya Delgado, P., Ruiz Sánchez de León, J. M. y Pedrero Pérez, E. (2012). Validación de un cuestionario de quejas cognitivas para adultos jóvenes: relación entre las quejas subjetivas de memoria, la sintomatología prefrontal y el estrés percibido. Revista de Neurología, 54, 137-150.

Luethi, M., Meier, B. y Sandi, C. (2009). Stress effects on working memory, explicit memory, and implicit memory for neutral and emotional stimuli in healthy men. Frontiers in Behavioural Neuroscience, 2 (5). (doi: 10.3389/neuro.08.005.2008).

Maruyama, T. (1997). Deficit of explicit memory in Parkinson's disease demonstrated by auditory-verbal and visual-design learning tasks. Nihon Rinsho, 55(1), 195-201.

Meichenbaum, D. y Cameron, R. (1987). Entrenamiento en inoculación de estrés: hacia un paradigma general para el entrenamiento en habilidades de afrontamiento. En D. Meichenbaum y M. E. Jaremko (Eds.), Prevención y reducción del estrés (pp. 139-156). Bilbao: Desclée de Brouwer.

Metternich, B., Schmidtke, K. y Hüll, M. (2009 May). How are memory complaints in functional memory disorder related to measures of affect, metamemory and cognition? Journal of Psychosomatic Research, 66(5), 435-44. Epub 2009 Mar 4.

Mimura, M. (2008). Memory impairment and awareness of memory deficits in early-stage Alzheimer's disease. The Tohoku Journal of Experimental Medicine, 215(2), 133-40.

Montejo, P., Montenegro, M., Fernandez, M. A. y Maestú, F. (2011). Subjective memory complaints in the elderly: Prevalence and influence of temporal orientation, depression and quality of life in a populationbased study in the city of Madrid. Aging and Mental Health, 15, 85-96.

Montejo, P., Montenegro, M., Fernández, M. A. y Maestú, F. (2012). Memory complaints in the elderly: Quality of life and daily living activities. A population based study. Archives of Gerontology and Geriatrics, 54, 298-304.

Muñoz, J. M. y Tirapu, J. (2004). Rehabilitación de las funciones ejecutivas. Revista de Neurología, 38, 656-63.

Nelson, T. O. (1996). Consciusness and metacognition. Am. Psychol, 51,10216. 
Nelson, T. O. y Narens, L. (1990). Metamemory: A theoretical framework and new findings. En G. Bower (Ed.), The psychology of learning and motivation (Vol. 26). New York: Academic Press.

Pearman, A., Storandt, M. (2004). Predictors of subjective memory in older adults. Journal of Gerontology: Psychological Sciences, 59, 4-6.

Pearman, A. (2009). Predictors of subjective memory in young adults. Journal of Adult Development, 16, 101-107.

Pedrero, E. J., Ruiz, J. M., Lozoya, P., Llanero, M., Rojo, G. y Puerta, C. (2011) Evaluación de los síntomas prefrontales: propiedades psicométricas y datos normativos del Cuestionario Disejecutivo (DEX) en una amplia muestra de población general española. Revista de Neurología, 52, 394-404.

Pedrero, E. J., Ruiz, J. M., Olivar, A., Bouso, J. C., Rojo, G, Llanero, M., et al. (2009). Versión española del Cuestionario Disejecutivo (DEX-Sp): propiedades psicométricas en adictos y población no clínica. Adicciones, 21, 155-166.

Pedrero, E. J. y Olivar, A. (2010). Estrés percibido en adictos a sustancias en tratamiento mediante la escala de Cohen: propiedades psicométricas y resultados de su aplicación. Anales de Psicología, 26, 302-309.

Pericot, I., Hernández, M., Lozano, M., Vilalta, J., Cruz, M. M. y López, S. (2009). Perfil cognitivo en la fibromialgia. Comparación con un grupo con deterioro cognitivo leve. Medicina Clínica,133(3), 91-4.

Pia, L, Neppi-Modona, M., Ricci, R., Berti, A. (2004). The anatomy of anosognosia for hemiplegia: a meta-analysis. Cortex, 40, 367-377.

Potter, G. G., Hartman, M. y Ward, T. (2009 Jul). Perceived stress and everyday memory complaints among older adult women. Anxiety Stress Coping, 22(4), 475-81.

Rey, A. (2009). Test de copia de una figura compleja. Madrid: TEA Ediciones.

Rosen, H.J. (2011). Anosognosia in neurodegenerative disease. Neurocase, 17, 231-241.

Ruiz, J. M., Fernández, S. y González, J. (2006). Aspectos teóricos actuales de la memoria a largo plazo: de las dicotomías a los continuos. Anales de Psicología, 22(2), 290-297.

Ruiz, J. M, Llanero, M, Lozoya, P., Fernández, M. A y Pedrero, E. (2010). Estudio neuropsicológico de adultos jóvenes con quejas subjetivas de memoria: implicación de las funciones ejecutivas y otra sintomatología frontal asociada. Revista de Neurolología, 51, 650-660.

Ruiz, J. M., Pedrero, E. J., Rojo, G., Llanero, M. y Puerta, C. (2011). Propuesta de un protocolo para la evaluación neuropsicológica de las adicciones. Revista de Neurología, 53, 483-93.

Schmand, B., Jonker, C., Hooijer, C. y Lindeboom, J. (1996). Subjective memory complaints may announce dementia. Neurology, 46, 121-125.
Schretlen, D., Bobholz, J. y Brandt, J. (1996). Development and psychometric properties of the Brief Test of Attention. The Clinical Neuropsychologist, 10, 80-89.

Schyner, D. M., Verfaellie, M., Alexander, M. P., LaFleche, G., Nicholls, L. y Kaszniak, A.W. (2004). A role for right medial prefrontal cortex in accurate feeling of knowing judgements: evidence from patients with lesions to frontal cortex. Neuropsychologia, 42, 957-966.

Sedó, M. A. (2007). Test de los cinco dígitos (FDT). Madrid: TEA.

Shallice, T. (1982). Specific impairments of planning. Philosophical Transactions of the Royal Society of London B, 298, 199-209.

Sunderland, A., Harris, J. y Baddeley, A. D. (1984). Assesing Everyday memory after severe head unjury. En Harris, J. F., Morris, P. E. (Eds.), Everyday memory, actions, and absent-mindedness (pp. 193-212). London: Academic Press.

Sunderland, A., Harris, J. E. y Gleave, J. (1984). Memory failures in everyday life following severe head injury. Journal of Clinical and Experimental Neuropsychology, 6, 127-142.

Tirapu, J. y Muñoz, J. M. (2005). Memoria y funciones ejecutivas. Revista de Neurología, 41, 475-484.

Verhaeghen, P., Geraerts, N. y Marcoen, A. (2000 Oct). Memory complaints, coping, and well-being in old age: a systemic approach. Gerontologist, 40(5), 540-8.

Verma, S. K., Pershad, D., Nehram R., Kaurm R. y Bhagatm K. (1996). Personality correlates of perceived memory disturbances. Journal of Personality and Clinical Studies, 12, 33-36.

Vernon, D., Egner, T., Cooper, N., Compton, T., Neilands, C., Sheri, A. y Gruzelier, J. (2003). The effect of training distinct neurofeedback protocols on aspects of cognitive performance. International Journal of Psychophysiology, 47, 75-85.

Vilkki, J., Servo, A. y Surma-Aho, O. (1999). Inaccurate prediction of retrieval in a face matrix learning task after right frontal lobe lesions. Neuropsychology, 13, 298-305.

Vilkki, J., Servo, A., Surma-Aho, O. (1998). Word list learning and prediction of recall after frontal lobe lesions. Neuropsychology, 12, 268-277.

Wechsler, D. (1999a). Escala de Inteligencia Wechsler para Adultos (WAIS-III) $\left(3^{\mathrm{a}}\right.$ Ed.). Madrid: TEA.

Wechsler, D. (1999b). Escala de Memoria Wechsler para Adultos (WMS-III) (3 Ed.). Madrid: TEA.

Wilson, B. A., Alderman, N., Burgess, P. W., Emslie, H., Evans, J. J. (1996). Behavioural assessment of the dysexecutive syndrome. Bury St. Edmunds, UK: Thames Valley Test.

(Artículo recibido: 17-2-2012; revisado: 8-5-2013; aceptado: 18-5-2013) 